\title{
Proceedings of the International Haemophilia Prophylaxis Study Group Meeting, November 2003, Montreal, PQ, Canada
}

\author{
B. M. FELDMAN, * P. BABYN, $\dagger$ A. S. DORIA, + L. HEIJNEN, $\mathbb{J}$ J. JACOBSON, R. KILCOYNE,** \\ B. LUNDIN, $\dagger \dagger$ M. MANCO-JOHNSON, + I M. MCLIMONT, $\mathbb{S}$ P. PETRINI, $\uparrow$ H. PETTERSSON*** \\ and V. S. BLANCHETTE $\dagger+\dagger$ ON BEHALF OF THE IPSG \\ *Associate Professor Pediatrics, Health Policy Management and Evaluation, and Public Health Sciences, University of \\ Toronto, Staff Rheumatologist, Hospital for Sick Children, Clinical Chief, Arthritis Team, Bloorview MacMillan \\ Children's Centre, Toronto, ON, Canada; †Radiologist-in-Chief, Department of Diagnostic Imaging, Hospital for Sick \\ Children, Toronto, ON, Canada; $\vdots$ Assistant Professor and Staff Radiologist, Department of Diagnostic Imaging, Hospital \\ for Sick Children, Toronto, ON, Canada; \$Consultant Physical Medicine and Rehabilitation Van Creveldkliniek, \\ University Medical Center Utrecht, Physiatrist and Medical Director Rehabilitation Center De Trappenberg, Huizen, The \\ Netherlands; Associate Professor, Department of Radiology, University of Michigan, Ann Arbour, MI, USA; **Professor \\ Emeritus, Department of Radiology, University of Colorado Health Sciences Center, Denver, CO, USA; ††Chief of Section \\ for Musculoskeletal Radiology, Department of Radiology, University Hospital of Lund, Lund, Sweden; $+\uparrow$ Professor, \\ Department of Pediatrics, Mountain States Regional Hemophilia and Thrombosis Center, University of Colorado Health \\ Sciences Centre, Denver, CO, USA; $\mathbb{S}$ P Population Health Sciences Research Program, Hospital for Sick Children, Toronto, \\ ON, Canada; $\uparrow$ Assistant Professor, Department of Pediatrics, Karolinska Hospital, Stockbolm, Sweden; ***Professor of \\ Radiology, Lund University, Sweden, Chief Medical Officer, Region of Scania; and †††Professor of Pediatrics, University \\ of Toronto, Chief, Division of Hematology/Oncology, Hospital for Sick Children, Toronto, ON, Canada
}

On 3rd and 4th November 2003, in Montreal Canada, the International Haemophilia Prophylaxis Study Group (IPSG) held its first scientific symposium. The theme of the symposium was assessment of joint damage in persons with haemophilia with an emphasis on the detection of early joint disease using newer physical assessment and imaging techniques such as magnetic resonance imaging (MRI). Progress of two expert working groups of the IPSG - the physical therapy (PT) and the MRI groups - was presented and discussed at the symposium. This paper is a brief report of the discussions that took place.

\section{The IPSG}

The IPSG was formed in 2001 by a group of interested expert clinicians and scientists. Membership of the IPSG and its expert working groups is

Correspondence: Dr Brian Feldman, Division of Rheumatology, 555 University Ave, Toronto, ON, Canada M5G 1X8.

Tel.: 416-813-5828; fax: 416-813-4989;

e-mail: brian.feldman@sickkids.ca

Accepted after revision 14 October 2004 detailed in the Appendix. The efforts of the IPSG are coordinated from The Hospital for Sick Children, Toronto, Canada; Ms Marjorie McLimont is the administrative coordinator. The purpose of the group is illustrated by its mission statement: 'To promote the generation and communication of new information regarding the administration and outcome of factor prophylaxis in individuals with haemophilia'. The focus of the IPSG has been to provide tools (for example, validated and standardized outcome measures) that will facilitate future clinical trials of haemophilia prophylaxis.

The IPSG has set a goal to hold annual meetings of invited experts and doctors involved in the care of persons with haemophilia. These meetings will focus on a specific theme or themes relevant to prophylaxis.

\section{Proceedings}

\section{The Physical Therapy Expert Working Group}

The first part of the meeting was devoted to discussions related to work undergone by the 
PT Expert Working Group. Dr Brian Feldman (Canada) presented recommendations from this group.

The PT Expert Working Group was charged with the task of advising the IPSG of the best methods for measuring joint health (for use in studies of prophylaxis). There are several methods for measuring the integrity of joints for children and adults with haemophilia. These methods include the World Federation of Haemophilia (WFH) Orthopedic Advisory Committee scale [1], and the more recent scales developed in Denver by Dr Manco-Johnson et al. [2] and in Stockholm by Dr Petrini working with the European Paediatric Network for Haemophilia Management (PedNet) [3]. The new scales were developed in response to perceived limitations in the older WFH scale; the WFH scale was never formally validated, it measures gross changes to joint structure and function but does not capture milder changes expected to occur with prophylaxis, and it does not account for normal physiological changes that occur in young children during development (e.g. changes in lower limb varus and valgus alignment that normally occur with growth). The newer scales were developed to be more sensitive to early arthropathy and to milder joint disease.

The PT Working Group had met previously - in November 2002 - and reviewed the newer scales. After review of the comparative strengths and weaknesses, the group resolved to unify the Denver and Stockholm scales into a single scale that incorporated the best features of both. The group hoped that having a single scale would improve the comparability of international studies looking at the prevention - or treatment - of haemophilia arthropathy. A tentative scale was drawn up, and then through an iterative process the scale was refined and a guidance document including definitions and procedures was developed.

To test the reliability of the new scale (titled the Haemophilia Joint Health Score, HJHS) a study was carried out in September 2003 at The Hospital for Sick Children, Toronto.

The purpose of the September study was to determine the inter-observer and the test-retest reliability of the new HJHS, as well as to determine the internal consistency of the included items. To do this, four physiotherapists - representing international experience - independently examined eight boys with severe haemophilia twice; each subject was examined at the same time of day, 1 day apart. The boys were selected from the Toronto clinic in order to represent a variety of ages and a wide spectrum of joint disease. The order of examination was randomly allocated.

It was found that the total score (the sum of all the items) of the HJHS had excellent inter-observer and test-retest reliability, and the internal consistency of the items - as measured by Cronbach's- $\alpha$ coefficient was also excellent. However, not all the items performed equally well.

The PT Expert Working Group identified some immediate next steps. They identified areas in the guidance document that needed clarification and items on the HJHS that needed improvement. The group will also work on developing a user-friendly work sheet that can be used to record joint findings in the clinical setting, and that will make scoring the HJHS easier.

Finally, the PT Expert Working Group has begun planning a larger study that will formally validate the HJHS. The validity study will ask the following questions:

1 What is the convergent and discriminant construct validity of the HJHS?

2 Can redundant or rarely endorsed items be removed from the HJHS?

Following the discussion of the HJHS PT score, Dr Lily Heijnen (the Netherlands) discussed the rationale for rehabilitative strategies, and their usefulness even in the era of primary factor prophylaxis.

Rehabilitation can be defined - in the setting of paediatrics - in terms of its goals of optimizing autonomy and social participation. Under the World Health Organizations recent International Classification of Functioning, Disability and Health (ICF) we can conceive of haemophilia joint disease as affecting the functional and structural integrity of the body, which in turn can limit normal activities and eventually restrict participation in desired life-events (e.g. sports, school and work).

Paediatric rehabilitation has a focus both on the child and on the family. Child-oriented rehabilitation focuses on normal development. Guided by Dynamic Systems theory, and Neuronal Group Selection theory, rehabilitation of children with haemophilia should not restrict activities, rather encourage normal behaviours - and therefore more normal development - except in situations where there is good evidence that restriction will do more good than harm. A family-centred approach is necessary to deal with the consequences of haemophilia on the family functioning and structure.

There are many lessons that rehabilitation health professionals can learn from animal studies of cartilage damage because of haemarthrosis. Direct 
damage from blood components as well as inflammatory and mechanical factors have been shown to play a part in joint damage from bleeding [4]. Most importantly, although:

1 Young children are likely to be more susceptible to blood induced damage than older children and adults.

2 A single bleed into a joint can produce changes that may induce permanent cartilage damage.

3 Joint damage includes an inflammatory component, but free radical induced degeneration is the dominant factor in damage.

4 Joint loading (bearing weight through the joint) during a bleed may be responsible for more damage than just bleeding alone.

Preventing joint damage, therefore, may include rehabilitation strategies as well as factor prophylaxis. Muscle weakness may, through imbalance around the joints, predispose to haemarthrosis and chronic synovitis. Imbalance may further lead to increasing deformity of the joints. Strengthening the muscles around joints may prevent abnormal loading and therefore further deformity, and better joint function may prevent bleeding. Furthermore, increasing fitness levels of children with haemophilia may increase the ability to participate in family activities and therefore lessen the impact of the disease on families. One might consider lifelong exercise and sport as a form of rehabilitative prophylaxis.

\section{The magnetic resonance imaging Expert Working Group}

The second half of the conference was devoted to sensitive imaging strategies that might be used in following persons treated with haemophilia prophylaxis. In parallel with the PT Expert Working Group, the MRI Expert Working Group was charged with the task of advising the IPSG of the best methods for imaging joints using MRI (for use in studies of prophylaxis). Dr Holger Pettersson (Sweden) provided a general overview of the state-of-the-art of imaging for haemophilic arthropathy.

Several modalities may be used to image the joints of children and adults with haemophilia. Different joint structures may benefit from imaging using these different modalities. Conventional radiographic X-ray and computerized tomography (CT) may be used to image haemosiderin in the joint and pseudotumours. MRI may be more sensitive to subtle and early lesions. Ultrasound (US) may have a role in imaging soft tissue structures.

Iron in haemosiderin has distinctive appearances depending on the imaging technique. For example, gross haemosiderin appears white on X-ray, and black on MRI.

Synovitis may best be imaged using MRI with contrast media. Several methods are available including 'dynamic' enhanced MRI. While dynamic MRI is difficult to apply, it may be a valuable method for following the waxing and waning course of synovitis and the progression to fibrosis.

Cartilage, also, can be imaged using different techniques. Plain X-rays can imply the state of the cartilage by the width of the joint space. A narrowed joint space suggests eroded cartilage. MRI can directly visualize cartilage and can differentiate between superficial and deep lesions.

Plain radiographs can provide a wealth of information about the state of juxta-articular bone in haemophilic arthropathy. Radiographs can demonstrate osteopenia, cysts, erosions, ankylosis and growth changes in a reasonably sensitive fashion. MRI may provide additional information (for example, regarding cartilage, soft tissues and bone); MRI can show bone marrow oedema even in the face of a normal bone radiograph. The significance of marrow oedema is not always clear, however. MRI can also demonstrate bone cysts, sometimes under intact cartilage that may be missed on plain X-rays.

Imaging plays several roles in the management of patients with haemophilia. In the clinical setting, imaging is often used to set goals and to guide surgical interventions. As research tools, imaging techniques are used both to make comparisons (i.e. to compare groups of patients treated differently) and for longitudinal follow-up.

Finally, to help guide both clinical and research applications, several groups have developed classification scoring systems. For example, plain X-rays may be quantified using the Pettersson or the ArnoldHillgartner scores. MRIs may be quantified using the European score or the Denver score.

Dr Bjorn Lundin (Sweden) discussed some of the work that the MRI Expert Group had done to create a 'Consensus' score that combined the best features of the European and Denver MRI systems.

The MRI Expert Working Group had a number of decisions to make while developing a consensus for MRI scales. The progressive Denver score [5] is simpler and quicker to use, while the additive European score $[3,6]$ is more detailed and meticulous. Progressive and additive scores differ not only in detail, but also in how they reflect the disease. With a progressive method the most severe finding determines the score, and with an additive method all findings influence the score. Thus, the progressive strategy stresses the 'quality' of the changes, and 
the additive strategy stresses the 'quantity' of the changes.

The group felt that if the Denver and the European scores could be modified and combined into a comprehensive system, they could be used in a complementary fashion.

Dr Andria Doria (Canada) presented the features of this consensus score, and the work that has been done by the MRI Working Group in establishing the reliability of the new MRI score.

The MRI has the potential to reveal much earlier effects of joint bleeding than conventional radiography. However, it is not known if minor changes predict long-term joint damage, or if they warrant special attention. The two scoring systems currently developed for reading MRI scans of haemophilic joints differ in their approach. The Denver system [5] uses a 'progressive' scoring algorithm in which the worst joint finding determines the score. Each joint can receive a score of $0-10$. The European system $[3,6]$ is an 'additive' ordinal scale. Each joint can receive a score of $0-28$ by adding up the abnormalities seen. More recently, the developers of both scores have met - as part of the IPSG - to form a combined 'Consensus' score. The combined score includes both progressive and additive elements.

The MRI Consensus had to take into account scoring in two broad domains:

1 Soft tissue

a Effusions/haemarthroses

b Synovial hyperplasia

c Haemosiderin deposition

2 Osteochondral tissues

a Erosions

b Subchondral cysts

c Cartilage loss

The purpose of the Consensus score is to be sensitive to early or subtle joint changes, such as those that might be seen in the setting of a clinical trial of primary prophylaxis. To this end features of the European score (better able to score and discriminate more severe changes) and the Denver score (more sensitive to early changes) were felt to be complimentary.

The MRI group met in Toronto, in March 2003, to carry out a reliability study of the new system. Weight-bearing MRI scans from the knees of 32 patients with haemophilia were collected. The subjects ranged from 4 to 16 years of age. Scans were collected from two participating countries (Sweden and Canada). Four radiologists independently scored each of the radiographs twice in a randomly determined order.
The inter- and intra-rater reliability of the Consensus score was very high. For example, the intraclass correlation coefficients for both the progressive and additive components were $>0.85$ between readers. Nonetheless, the group identified areas of improvement that they will work on while developing a definitive Consensus scoring system.

At the close of the meeting, Dr Jon Jacobson (USA) presented a 'state-of-the-art' discussion of the use of sonography in imaging the joints of persons with haemophilia.

Ultrasound technology has progressed to the point that it may take on an exciting role in the evaluation of musculoskeletal problems in haemophilia. Portable US equipment (the size of a laptop computer) is now available at reasonable price. Probes that provide high resolution (high frequency) or that can image at great depth (low frequency) are available.

Different features of anatomy can be seen using US techniques. Tendons are visualized as hyperechoic fibrillar structures. Muscle is hypoechoic and speckled. Bone can be seen as hyperechoic with shadowing. Different types of cartilage have different US appearances; fibrocartilage is hyperechoic and hyaline cartilage is hypoechoic. When imaging the joint, joint fluid may be seen as hyper- or hypoechoic (or even as anechoic if it is a simple fluid). Synovitis therefore may need additional techniques-like colour and power Doppler imaging - in order to detect hypervascularity.

The US can be used to image joint abnormalities seen in haemophilia. The joint recess can reveal signs of haemorrhage and effusion as well as synovitis. As discussed above, colour and power Doppler, or flow techniques can be used to differentiate these conditions. When imaging cartilage, bone destruction, or the changes of osteoarthritis that are frequently seen in advanced haemophilic arthropathy, US may have some limitations. Given the nature of US probes prominences can be imaged well, whereas indentations can be difficult to see. Despite this, it has been recently demonstrated that in rheumatoid arthritis, US may be even more sensitive than plain X-ray at determining bone erosions.

The US is very good at imaging soft tissue structures. The technique can be used to detect heterotopic ossification, bursal haemorrhage, intermuscular bleeds, and US is an excellent way to detect i.m. bleeds (e.g. iliopsoas bleeds).

Given these strengths, US may have a large beneficial role in the management of haemophilia. It may be an excellent way to detect involvement of structures adjacent to bleeding areas, for example, 
nerve compression by haematomas may be detected in this fashion. US can be used both to detect and quantitate acute haemarthroses, and to follow more chronic changes-like synovitis and cartilage erosions. US may be used to anatomically localize bleeds that are clinically difficult to place. Finally, US may be used to follow patients in the setting of clinical trials; US can be used to detect early bleeds and to follow the effects of early treatment on the bleeding pattern.

\section{Conclusion}

Haemophilia prophylaxis - as the emerging worldwide standard of care - has posed new challenges. The previously used imaging and examination methods may no longer be sensitive enough to assess milder, but still clinically important, arthropathy. The International Prophylaxis Study Group's PT and MRI Expert Working Groups have developed reliable assessment tools that have combined the best features of previous instruments. Further work is now proceeding to establish the validity and measurement properties of both the HJHS, and the Consensus MRI score so that they can be used in future clinical studies.

\section{Acknowledgements}

Funding support for the activities of the IPSG is provided by grants from Bayer Health Care Biological Products Division, Baxter BioScience, Novo Nordisk, Wyeth Pharmaceuticals, and ZLB Behring that are administered through the Hospital for Sick Children Foundation, Toronto, Canada.

\section{References}

1 Gilbert MS. Prophylaxis: musculoskeletal evaluation. Semin Hematol 1993; 30 (3 Suppl. 2): 3-6.

2 Manco-Johnson MJ, Nuss R, Funk S, Murphy J. Joint evaluation instruments for children and adults with haemophilia. Haemophilia 2000; 6: 649-57.

3 Hill F, Ljung R. Meeting Report - third and fourth workshops of the European Paediatric Network for Haemophilia Management. Haemophilia 2003; 9: 223-8.

4 Roosendaal G, Lafeber FP. Blood-induced joint damage in haemophilia. Semin Thromb Hemost 2003; 29: 3742.

5 Nuss R, Kilcoyne R, Geraghty $S$ et al. MRI findings in haemophilic joints treated with radiosynoviorthesis with development of an MRI scale of joint damage. Haemophilia 2000; 6: 162-9.
6 Lundin B, Pettersson H, Ljung R. A new magnetic resonance imaging scoring method for assessment of haemophilic arthropathy. Haemophilia 2004; 10: 383-9.

\section{Appendix}

The IPSG membership consists of a steering committee and invited experts. The steering committee members are:

1 Dr Victor Blanchette (Canada), Chair

2 Dr Louis Aledort (USA), Co-chair

3 Dr Rolf Ljung (Sweden), Co-chair

4 Dr Brian Feldman (Canada)

5 Dr Alessandro Gringeri (Italy)

6 Dr Marilyn Manco-Johnson (USA)

7 Dr Pia Petrini (Sweden)

8 Dr Georges Rivard (Canada)

9 Dr Wolfgang Schramm (Germany)

10 Dr Marijke van den Berg (the Netherlands)

Several subcommittees (expert working groups) have been formed. These include:

\section{The MRI Expert Working Group}

1 Dr Holger Pettersson (Sweden), Chair

2 Dr Paul Babyn (Canada)

3 Dr Andrea Doria (Canada)

4 Dr Ray Kilcoyne (USA)

5 Dr Björn Lundin (Sweden)

6 Dr Rachelle Nuss (USA)

7 Dr Georges Rivard (Canada)

\section{The Physical Therapy Expert Working Group}

1 Dr Marilyn Manco-Johnson (USA), Co-chair

2 Dr Pia Petrini (Sweden), Co-chair

3 Ms Britt-Marie Bergstrom (Sweden)

4 Dr Brian Feldman (Canada)

5 Ms Sharon Funk (USA)

6 Ms Pamela Hilliard (Canada)

7 Dr Marijke van den Berg (the Netherlands)

8 Mr Nick Zourikian (Canada)

\section{The Outcome Measures Expert Working Group}

1 Dr Wolfgang Schramm (Germany), Co-chair

2 Dr Victor Blanchette (Canada), Co-chair

3 Dr Louis Aledort (USA)

4 Dr Rhonda Bohn (USA)

5 Dr Monika Bullinger (Germany)

6 Dr Brian Feldman (Canada)

7 Dr Alessandro Gringeri (Italy)

8 Dr Hilary Llewellyn-Thomas (USA) 
9 Dr Lorenzo Mantovani (Italy)

10 Dr Alex Miners (UK)

11 Dr Marijke van den Berg (the Netherlands)
12 Dr Ben van Hout (the Netherlands)

13 Dr Sylvia von Mackensen (Germany)

14 Dr Nancy Young (Canada) 